CARDIOVASCULAR MEDICINE

\title{
Wine drinking is associated with increased heart rate variability in women with coronary heart disease
}

\author{
I Janszky, M Ericson, M Blom, A Georgiades, J-O Magnusson, H Alinagizadeh, S Ahnve
}

Heart 2005:91:314-318. doi: 10.1136/hrt.2004.035105

See end of article for authors' affiliations .......................

Correspondence to: Professor Staffan Ahnve, Preventive Medicine, Karolinska Institute, Norrbacka, 6th floor, Karolinska Hospital, 'SE17176 Stockholm, Sweden; staffan.ahnve@ medhs.ki.se

Received 28 January 2004

\begin{abstract}
Objective: To test the hypothesis that alcohol consumption is positively related to heart rate variability (HRV) in women with coronary heart disease (CHD) and therefore that cardiac autonomic activity is potentially implicated in the mediation of the favourable effects of moderate drinking.

Design, settings, and patients: Cross sectional study of female patients who survived hospitalisation for acute myocardial infarction or underwent a revascularisation procedure, percutaneous transluminal coronary angioplasty, or coronary artery bypass grafting.

Main outcome measures: Ambulatory 24 hour ECG was recorded during normal activities. The mean of the standard deviations of all normal to normal intervals for all five minute segments of the entire recording (SDNNI) and the following frequency domain parameters were assessed: total power, high frequency power, low frequency power, and very low frequency power. A standardised questionnaire evaluated self reported consumption of individual alcoholic beverage types: beer, wine, and spirits. Other clinical characteristics, such as age, body mass index, smoking habits, history of diabetes mellitus, menopausal status, educational status, and treatment, were also assessed.

Results: Wine intake was associated with increased HRV in both time and frequency domains independently of other clinical covariates (for example, In SDNNI was 3.89 among wine drinkers $v 3.59$ among wine non-drinkers in the multivariate model; $p=0.014$ ). In contrast, consumption of beer and spirits and the total amount of alcohol consumed did not relate significantly to any of the HRV parameters. Conclusion: Intake of wine, but not of spirits or beer, is positively and independently associated with HRV in women with CHD. These results may contribute to the understanding of the complex relation between alcohol consumption and $\mathrm{CHD}$.
\end{abstract}

C ompelling epidemiological evidence suggests that moderate intake of alcoholic beverages, especially wine, is associated with lower risk of coronary heart disease (CHD) morbidity and mortality. ${ }^{1-4}$ Furthermore, moderate alcohol intake is related to reduced mortality after acute myocardial infarction (AMI). ${ }^{5}$ Mechanisms of this favourable effect of alcoholic beverages are not entirely clear; however, they may include increased high density lipoprotein cholesterol concentrations, ${ }^{7}$ improved coagulation profile, ${ }^{89}$ lower levels of inflammation, ${ }^{10}$ greater insulin sensitivity, ${ }^{11}$ better endothelial function, ${ }^{12}$ reduced endothelin 1 synthesis, ${ }^{13}$ low density lipoprotein oxidation, ${ }^{14}$ and smooth muscle proliferation. ${ }^{15}{ }^{16}$

Heart rate variability (HRV), the amount of fluctuation of the beat to beat differences, is known to be a reliable, noninvasive marker of autonomic nervous system activity. Decrease in HRV has been found to predict CHD morbidity and mortality in apparently healthy populations ${ }^{17}{ }^{18}$ and in patients after an acute coronary event. ${ }^{19-23}$

Relatively few studies have examined the associations between drinking habits and HRV parameters, and the results are conflicting. ${ }^{24-29}$ The purpose of this study was to test the hypothesis that alcohol consumption is positively related to HRV in CHD and therefore that cardiac autonomic activity is potentially implicated in mediating the favourable effects of moderate drinking. We assessed total alcohol consumption, consumption of different types of alcoholic beverages, and time and frequency domain parameters of HRV by using ambulatory ECG monitoring in women with CHD.

\section{METHODS}

\section{Study population}

One hundred and two women, who survived hospitalisation for AMI or underwent a revascularisation procedure, percutaneous transluminal coronary angioplasty (PTCA), or coronary artery bypass grafting (CABG) at Huddinge University Hospital or St Göran's Hospital, Stockholm, Sweden, were enrolled. We based the diagnosis of AMI on World Health Organization criteria of typical enzyme patterns and chest pain or diagnostic ECG changes. Independently of the present report patients participated in a randomised controlled intervention trial. Consecutively, all eligible women below 75 years were asked to participate in the trial and those who agreed to do so were randomly assigned to either the control or the intervention group. The intervention comprised a stress management program specifically designed for women with CHD and targeted at improving social functioning, self esteem, and coping and decreasing depression and type A behaviour. ${ }^{30}$

\section{Ambulatory ECG monitoring and analyses of HRV}

One year and five months ( \pm 2.5 months) after randomisation a two channel ECG (Spacelab 90205; Spacelab Inc, Redmond, Washington, USA) was recorded over 24 hours during normal daily activities. The ECG electrodes were attached at the position of CM-V5 (left anterior axillary line sixth rib) and CS-Vl (fourth rib at the sternal border). Patients were asked to continue their usual medications.

The 24 hour Holter tape recordings were digitised and QRS labelled by a commercially available PC based system (Aspect Holter System, Daltek, Borlänge, Sweden). Arrhythmias were

\footnotetext{
Abbreviations: $A M I$, acute myocardial infarction; $C A B G$, coronary artery bypass grafting; CHD, coronary heart disease; HRV, heart rate variability; PTCA, percutaneous transluminal coronary angioplasty; SDNNI, mean of the standard deviations of all normal to normal intervals for all five minute segments of the entire recording
} 
automatically analysed and the QRS complexes classified. Consecutive RR intervals were expressed in centiseconds and analysed in units of five minute epochs by custom software. To be accepted for additional analysis, we required at least $96 \%$ of the QRS complexes to be classified as normal by the Aspect system. ${ }^{31}$ The time series of $R R$ intervals were resampled at a frequency of two samples a second. Gaps in the time series due to non-normal RR intervals (QRS labelled by the Aspect System classification as noise or extrasystoles) were filled with values calculated by linear interpolation between adjacent normal RR intervals. Misclassified dropped beats deviating more than $3.0 \mathrm{SD}$ from the normal $\mathrm{RR}$ interval of each epoch were also automatically checked.

Both time and frequency domain measures were analysed. The mean of the standard deviations of all normal to normal intervals for all five minute segments of the entire recording (SDNNI, ${ }^{32}$ in $\mathrm{ms}$ ) was obtained from the time series of normal RR intervals. Frequency domain parameters were calculated by an autoregressive method as described previously $^{33}$ : high frequency power, $0.15-0.40 \mathrm{~Hz}$; low frequency power, 0.04-0.15 Hz; very low frequency power, 0.0033$0.04 \mathrm{~Hz}$; and total power (in $\mathrm{ms}^{2}$ ).

Patients were not enrolled if they had non-sinus rhythm or if less than $50 \%$ of the original ECG recording was available for analysis.

\section{Evaluation of alcohol consumption}

Consumption of alcoholic beverages was assessed by the corresponding items of the Willett food frequency questionnaire, ${ }^{34}$ which has shown excellent correlation with alcohol consumption as measured by four diet records of one week each (obtained 3-4 months apart) in a Swedish female cohort. ${ }^{35}$ The questionnaire asks about the usual frequency and quantity of intake of five beverage types: regular beer, strong beer, wine, light spirits (for example, liqueur, vermouth, and port), and spirits. The estimated alcohol content of these beverages was 3.5\%, $4.9 \%, 11 \%, 19 \%$, and $39 \%$, respectively. Average daily alcohol intake was calculated in grams. Daily consumption of alcohol was categorised as follows: 0 (abstainers), $0.1-5.0 \mathrm{~g}$ /day (light drinkers: up to half a standard drink a day), and > $5.0 \mathrm{~g} /$ day (moderate drinkers: over half a standard drink a day). Daily consumption of alcohol from regular beer, strong beer, wine, light spirits, and spirits was also calculated. Because overall consumption of strong beer and light spirits was low (mean intake 0.14 and $0.22 \mathrm{~g} /$ day, respectively), we grouped these beverages with regular beer and regular spirits, respectively, in beverage specific analyses. Binomial categorical variables were created to indicate whether patients consumed beer, wine, or spirits at all.

\section{Other covariates}

Educational attainment was classified into three levels: mandatory schooling only, completion of high school, and some college. Menopausal status was categorised as premenopausal, postmenopausal, taking hormone replacement therapy, and postmenopausal without hormone replacement therapy. Smoking status was categorised as never, current, or former smoking. Clinical history of diabetes mellitus was assessed. Height and weight were measured and body mass index was calculated.

\section{Statistical analysis}

HRV parameters were logarithmically transformed, as their distribution was skewed. Student's $t$ test was used to detect significance of differences between continuous variables for two groups; one way analysis of variance was applied in the case of three groups. Categorical data were compared by $\chi^{2}$ test.
Table 1 Clinical characteristics of the study group ( $\mathrm{n}=102$ )

\begin{tabular}{ll}
\hline Mean alcohol consumption (g/day) & $1.62(2.57)$ \\
Mean consumption of & \\
Beer (g/day) & $0.61(1.19)$ \\
Wine (g/day) & $0.53(1.16)$ \\
Spirits (g/day) & $0.48(1.11)$ \\
Heart rate variability & \\
In SDNNI & $3.81(0.52)$ \\
In total power & $7.14(1.16)$ \\
In VLF power & $6.36(1.06)$ \\
In LF power & $5.85(1.23)$ \\
In HF power & $5.62(1.35)$ \\
Mean RR (ms) & $897(130)$ \\
Age (years) & $64.3(8.1)$ \\
Body mass index (kg/m ${ }^{2}$ ) & $26.5(4.3)$ \\
Smoking & $7.8 \%$ \\
Current & $48.0 \%$ \\
Former & $44.1 \%$ \\
Never & $20.6 \%$ \\
Diabetes mellitus & \\
Menopausal status & $8.9 \%$ \\
Premenopausal & $17.8 \%$ \\
Postmenopausal on HRT & $73.3 \%$ \\
Postmenopausal without HRT & \\
Educational attainment & $68.6 \%$ \\
Mandatory & $17.6 \%$ \\
High school & $13.7 \%$ \\
College or university & \\
Medication use & $90.2 \%$ \\
Aspirin & $24.5 \%$ \\
Calcium channel blocker & $78.4 \%$ \\
$\beta$ Blocker & $26.5 \%$ \\
ACE inhibitor & $77.5 \%$ \\
Statin & \\
Index event* & $35.1 \%$ \\
AMl & $38.2 \%$ \\
CABG & $36.3 \%$ \\
PTCA & \\
Stress management & \\
\hline Continuous data are presented as mean (SD). \\
ACE, angiotensin converting enzyme; AMl, acute myocardial infarction; \\
CABG, coronary artery bypass grafting; HF, high frequency power; HRT, \\
hormone replacement therapy; LF, low frequency power; PTCA, \\
percutaneous transluminal coronary angioplasty; SDNNI, mean of \\
standard deviations of all normal to normal intervals for all five minute \\
segments of the entire recording; VLF, very low frequency power. \\
\end{tabular}

After adjustment for potentially confounding factors, least squares mean (SE) was calculated according to the general linear model for the parameters of HRV in each category of alcoholic beverage. Potential confounders were age, menopausal status, body mass index, smoking habits, educational status, history of diabetes mellitus, and treatment status (intervention versus control). In addition we adjusted for medications. Stratified analyses assessed possible effect modification. SAS version 8.2 (SAS Institute, Cary, North Carolina, USA) and SPSS version 11.0 for Windows (SPSS Inc, Chicago, Illinois, USA) were used for statistical analyses.

The study was approved by the ethics committee of Karolinska Institute at Huddinge University Hospital. Informed consent was obtained from all patients.

\section{RESULTS}

Table 1 presents the distribution of the study variables among women with complete data on HRV measurements and alcohol intake $(\mathrm{n}=102)$.

\section{HRV, heart rate, and alcohol consumption}

Table 2 presents the HRV parameters and mean RR interval in the group of abstainers and light $(0.1-5.0 \mathrm{~g} /$ day $)$ and moderate ( $>5.0 \mathrm{~g} /$ day) drinkers before and after adjustment. Although HRV parameters appeared to be higher with increasing alcohol intake, we found no significant 
Table 2 Heart rate variability parameters and mean RR interval according to daily alcohol consumption

\begin{tabular}{|c|c|c|c|c|}
\hline & \multicolumn{3}{|c|}{ Daily alcohol consumption (g/day) } & \multirow[b]{2}{*}{ p Value } \\
\hline & $0(n=20)$ & $0.1-5.0(n=76)$ & $>5.0(n=6)$ & \\
\hline \multicolumn{5}{|l|}{ In SDNNI } \\
\hline Unadjusted & $3.68(0.11)$ & $3.83(0.06)$ & $3.91(0.38)$ & 0.48 \\
\hline Adjusted* & $3.65(0.18)$ & $3.83(0.13)$ & $3.89(0.25)$ & 0.44 \\
\hline \multicolumn{5}{|l|}{ In total power } \\
\hline Unadjusted & $6.90(0.23)$ & $7.19(0.13)$ & $7.31(0.83)$ & 0.57 \\
\hline Adjusted & $6.87(0.39)$ & $7.20(0.28)$ & $7.31(0.56)$ & 0.54 \\
\hline \multicolumn{5}{|l|}{ In VLF power } \\
\hline Unadjusted & $6.19(0.21)$ & $6.39(0.12)$ & $6.49(0.63)$ & 0.73 \\
\hline Adjusted & $5.99(0.36)$ & $6.27(0.26)$ & $6.41(0.51)$ & 0.58 \\
\hline \multicolumn{5}{|l|}{ In LF power } \\
\hline Unadjusted & $5.57(0.25)$ & $5.91(0.13)$ & $6.06(0.94)$ & 0.50 \\
\hline Adjusted & $5.59(0.42)$ & $5.95(0.30)$ & $6.08(0.59)$ & 0.54 \\
\hline \multicolumn{5}{|l|}{ In HF power } \\
\hline Unadjusted & $5.33(0.26)$ & $5.68(0.15)$ & $5.72(0.99)$ & 0.57 \\
\hline Adjusted & $5.47(0.45)$ & $5.85(0.33)$ & $5.79(0.65)$ & 0.58 \\
\hline \multicolumn{5}{|l|}{ Mean RR } \\
\hline Unadjusted & $884(28)$ & $912(14)$ & $775(51)$ & 0.04 \\
\hline Adjusted & 850 (39) & 872 (28) & 730 (55) & 0.03 \\
\hline
\end{tabular}

Data are mean (SE)

${ }^{*}$ Adjusted for age, menopausal status, body mass index, smoking habits, educational status, history of diabetes mellitus, and treatment status (intervention $v$ control).

differences. However, mean RR interval was significantly differently distributed between the alcohol consumption categories; the highest values were registered among light drinkers.

HRV and consumption of specific alcoholic beverages Table 3 presents the HRV parameters and mean RR interval according to the consumption of the three beverage types. Most of the HRV measures were significantly higher among women drinking wine in the unadjusted as well as in the adjusted models. HRV parameters also tended to be higher among beer drinkers and spirit drinkers than among women who did not drink beer or spirits, but these differences did not reach the level of significance. These findings remained essentially unchanged after further adjustment for $\beta$ blockers and calcium channel blockers (ln SDNNI among wine drinkers and wine non-drinkers was $3.89 v 3.59$, respectively, in the model with further adjustment for medication, $\mathrm{p}=0.019)$. Although mean RR interval was longer among wine drinkers $(\mathrm{p}=0.026$ and $\mathrm{p}=0.064$ for the unadjusted and adjusted models, respectively), adjusting for mean RR interval when testing the relation between wine drinking and HRV parameters gave similar results (for example, In SDNNI was $3.92 v 3.68, \mathrm{p}=0.043$ in the model with further adjustment for mean RR). Furthermore, we examined maximum heart rate as an indicator for physical activity during the 24 hour recording period and found no relation with wine intake and virtually no effect of adjustment.

To evaluate in more detail the relation of HRV to specific beverage intake, we also categorised consumption of beverage types as non-drinking, drinking below the median, and drinking above the median. In SDNNI was 3.58 versus 3.96 versus $3.80(\mathrm{p}=0.025$, adjusted model $)$ in these three groups of wine drinking, respectively. The other HRV

Table 3 Heart rate variability parameters and mean RR interval according to consumption of wine, beer, and spirits

\begin{tabular}{|c|c|c|c|c|c|c|c|c|c|}
\hline & \multicolumn{9}{|c|}{ Daily consumption of alcoholic beverages (g/day) } \\
\hline & \multicolumn{3}{|l|}{ Wine } & \multicolumn{3}{|l|}{ Beer } & \multicolumn{3}{|l|}{ Spirits } \\
\hline & Yes $(n=69)$ & No $(n=33)$ & p Value & Yes $(n=52)$ & No $(n=50)$ & p Value & Yes $(n=54)$ & No $(n=48)$ & p Value \\
\hline \multicolumn{10}{|l|}{ In SDNNI } \\
\hline Unadjusted & $3.88(0.07)$ & $3.65(0.07)$ & 0.025 & $3.87(0.07)$ & $3.74(0.07)$ & 0.07 & $3.86(0.07)$ & $3.74(0.07)$ & 0.16 \\
\hline Adjusted ${ }^{*}$ & $3.89(0.12)$ & $3.59(0.15)$ & 0.014 & $3.87(0.14)$ & $3.76(0.13)$ & 0.30 & $3.88(0.14)$ & $3.74(0.13)$ & 0.21 \\
\hline \multicolumn{10}{|l|}{ In total power } \\
\hline Unadjusted & $7.30(0.15)$ & $6.81(0.16)$ & 0.049 & $7.29(0.16)$ & $6.99(0.16)$ & 0.10 & $7.24(0.17)$ & $7.03(0.16)$ & 0.36 \\
\hline Adjusted & $7.33(0.28)$ & $6.72(0.33)$ & 0.023 & $7.31(0.31)$ & $7.06(0.29)$ & 0.31 & $7.30(0.30)$ & $7.05(0.30)$ & 0.31 \\
\hline \multicolumn{10}{|l|}{ In VLF power } \\
\hline Unadjusted & $6.50(0.14)$ & $6.06(0.14)$ & 0.062 & $6.43(0.14)$ & $6.28(0.15)$ & 0.24 & $6.46(0.15)$ & $6.24(0.15)$ & 0.18 \\
\hline Adjusted & $6.41(0.25)$ & $5.80(0.30)$ & 0.014 & $6.30(0.28)$ & $6.21(0.27)$ & 0.66 & $6.40(0.28)$ & $6.11(0.27)$ & 0.22 \\
\hline \multicolumn{10}{|l|}{ In LF power } \\
\hline Unadjusted & $6.02(0.16)$ & $5.49(0.16)$ & 0.035 & $6.02(0.17)$ & $5.67(0.17)$ & 0.07 & $5.98(0.18)$ & $5.70(0.17)$ & 0.32 \\
\hline Adjusted & $6.09(0.29)$ & $5.44(0.35)$ & 0.024 & $6.08(0.32)$ & $5.79(0.31)$ & 0.25 & $5.77(0.32)$ & $6.07(0.32)$ & 0.27 \\
\hline \multicolumn{10}{|l|}{ In HF power } \\
\hline Unadjusted & $5.78(0.17)$ & $5.27(0.19)$ & 0.089 & $5.84(0.19)$ & $5.38(0.19)$ & 0.06 & $5.67(0.19)$ & $5.56(0.18)$ & 0.75 \\
\hline Adjusted & $5.96(0.32)$ & $5.37(0.39)$ & 0.059 & $6.05(0.35)$ & $5.61(0.34)$ & 0.12 & $5.89(0.35)$ & $5.72(0.35)$ & 0.56 \\
\hline \multicolumn{10}{|l|}{ Mean RR } \\
\hline Unadjusted & 918 (15) & $857(22)$ & 0.026 & $906(18)$ & $890(18)$ & 0.51 & 891 (18) & 906 (19) & 0.55 \\
\hline Adjusted & 872 (29) & 821 (34) & 0.064 & 871 (31) & 849 (30) & 0.39 & 852 (31) & $864(31)$ & 0.63 \\
\hline
\end{tabular}

Data are mean (SE).

*Adjusted for age, menopausal status, body mass index, smoking habits, educational status, history of diabetes mellitus, and treatment status (intervention $v$ control). 
parameters also differed significantly between the three groups, except for high frequency power, which was only marginally significant. However, there were no significant differences in HRV measures between the corresponding categories of consumption of spirits or beer.

Furthermore, we performed stratified analyses in selected clinical subgroups to ensure that our results were consistent. The results were not materially different when analyses were restricted to the control or to the intervention group, to patients $\geqslant 65$ years or to patients $<65$ years old, or to patients with AMI or patients who underwent CABG or PTCA.

We also tested the hypothesis that patients drinking beer or spirits may have a trend for higher HRV only due to their higher prevalence of wine drinking. When we simultaneously controlled for intake of other beverage types, wine intake remained significantly associated with HRV (ln SDNNI among wine drinkers was 3.61 and among non-drinkers, 3.89; $\mathrm{p}=0.041$, adjusted model). However, the tendency towards higher HRV among the consumers of beer and spirits was considerably attenuated in the simultaneous analyses (ln SDNNI $3.76 \vee 3.74, p=0.85$, and $3.76 \vee 3.73, p=0.86$, for drinkers versus non-drinkers of beer and spirits, respectively).

\section{DISCUSSION}

In this study of women with CHD we found that wine intake was associated with increased HRV independently of the potential confounding factors and of the intake of other beverages. In contrast, consumption of beer and spirits or the total amount of alcohol consumed did not relate significantly to any of the HRV parameters.

Convincing evidence suggests that alcohol consumption at moderate levels decreases CHD morbidity and mortality. ${ }^{1-4}$ Moreover, recent studies found lower mortality after myocardial infarction among patients with moderate alcohol intake. $^{5} 6$

Why moderate alcohol intake is associated inversely with CHD is not completely clear. A positive effect of alcohol on lipid profile is consistently reported. For instance, Langer and colleagues ${ }^{7}$ claimed that about half of the observed protection against CHD afforded by moderate alcohol consumption is mediated by an increase in high density lipoprotein. Other candidates for the favourable effect are decreased fibrinogen, ${ }^{8} 9$ decreased platelet activity, ${ }^{36}$ lower levels of inflammation, $^{10}$ greater insulin sensitivity, ${ }^{11}$ better endothelial function, ${ }^{12}$ reduced endothelin 1 synthesis, ${ }^{13}$ reduced low density lipoprotein oxidation, ${ }^{14}$ and smooth muscle proliferation. ${ }^{15}{ }^{16}$ Given the compelling evidence that decreased HRV is an independent predictor of CHD mortality, ${ }^{17-23}$ the present findings suggest that an increase in HRV may be a potential pathway for the protective effect of moderate alcohol consumption on CHD.

Whether the effect of moderate alcohol consumption on CHD is attributable to ethanol alone or if there are beverage specific differences is debated. In a recent review Gronbaek ${ }^{37}$ concluded that wine drinkers are at a decreased risk of death from cardiovascular disease compared with non-wine drinkers, whereas other meta-analyses found that wine drinking confers no particular benefit. ${ }^{38}{ }^{39}$ However, in this study wine was the only independent determinant of HRV among the alcoholic beverages, suggesting that only wine may have favourable effects mediated by this pathway.

\section{Comparison with previous studies}

According to previous observations acute alcohol intake decreases HRV, especially the indexes of vagal activity. ${ }^{40-43}$ It has also been shown that chronic excessive intake of alcohol is associated with decreased HRV. ${ }^{44}{ }^{45}$
However, studies on usual daily alcohol consumption and HRV found varying and apparently opposite effects. Ryan and Howes ${ }^{27}$ reported a negative relation between HRV and usual alcohol intake in apparently healthy men. In the Framingham study, Tsuji and colleagues ${ }^{25}$ found a positive association between HRV and alcohol intake, but the association was no longer significant after controlling for potential determinants of HRV. In line with this finding alcohol was not a significant independent predictor in the studies of Virtanen and colleagues ${ }^{28}$ and Stolarz and associates. ${ }^{29}$ Nevertheless, according to Kupari and colleagues, ${ }^{24}$ alcohol consumption is positively related to HRV in multivariate models in women, but not in men. All these studies examined apparently healthy people or population samples, except for the investigation of Virtanen and colleagues, ${ }^{28}$ where patients with newly diagnosed hypertension were studied in addition to healthy volunteers.

Christensen and colleagues, ${ }^{26}$ in a study population similar to ours, found that consumption of wine, but not beer, was associated with higher HRV among patients referred for coronary angiography. However, this effect was not independent of consumption of polyunsaturated fatty acids derived from fish, which was strongly related to HRV.

\section{Study limitations}

Several potential limitations of our study need to be considered, such as the use of self reported assessment of alcohol intake, which may lead to an underestimation of the real intake. However, there is no reason to believe that patients with lower HRV would underestimate their alcohol consumption to a greater extent.

The validity of our findings may be limited by the design of our study. As with any observational study, unevenly distributed characteristics associated with the amount of HRV may lead to overestimation or underestimation of the true effect of alcohol consumption. Multivariate adjustment here did not attenuate the association between wine intake and HRV. However, we cannot exclude the possibility of residual confounding, for example, by the effect of polyunsaturated fatty acids, which was a serious confounder in the study by Christensen and colleagues. ${ }^{26}$ At the same time, a remaining confounder would need to be associated with both alcohol consumption and HRV and generally unrelated to the factors considered in our multivariate analyses.

The sample size may not have been sufficient in this study to detect an association between HRV and consumption of beer or spirits or total amount of alcohol consumed. Nevertheless, the association with wine intake was already significant at this level of statistical power, and the simultaneous analyses of the beverages types showed virtually no tendency for higher HRV among beer or spirit consumers.

Another potential limitation of the present study is the possibility that some women ceased drinking in response to the severity of their illness. Since we have no information on drinking habits in the past, the "sick quitter" hypothesis ${ }^{46}$ may provide an alternative explanation for our findings.

We investigated women with established CHD and our findings are most readily generalised to similar populations. Women differ from men in drinking habits and alcohol metabolism, and recent studies suggest that wine drinking may have stronger effects on atherosclerosis in women. ${ }^{47-49} \mathrm{In}$ addition, as alcohol consumption was rather modest among our patients we cannot extrapolate our results to heavier alcohol consumption.

\section{Conclusion}

Intake of wine, but not spirits or beer, was positively and independently associated with HRV in women with CHD. Our 
results, suggesting that HRV may be an important linking factor, may contribute to the understanding of the complex relation of alcohol consumption with CHD.

\section{ACKNOWLEDGEMENTS}

This study was supported by grants form the Ansgarius Foundation, the Belvén Foundation, the Swedish Heart and Lung Foundation, the Public Health Committee and Expo-95 of Stockholm County Council, the Swedish Medical Research Council (project 19X-11629), and the Vardal Foundation, Stockholm, Sweden.

\section{Authors' affiliations}

I Janszky, M Blom, A Georgiades, H Alinagizadeh, S Ahnve, Preventive

Medicine, Department of Public Health Sciences, Karolinska Institute, and Centre of Public Health, Stockholm County Council, Stockholm, Sweden

M Ericson, Department of Industrial Economics, Royal Institute of Technology, Stockholm, Sweden

J-O Magnusson, Department of Cardiology, St Göran's Hospital, Stockholm, Sweden

Conflicts of interest: none.

\section{REFERENCES}

1 Maclure M. Demonstration of deductive meta-analysis: ethanol intake and risk of myocardial infarction. Epidemiol Rev 1993;15:328-51.

2 St Leger AS, Cochrane AL, Moore F. Factors associated with cardiac mortality in developed countries with particular reference to the consumption of wine. Lancet 1979:i: 1017-20.

3 Marmot MG, Rose G, Shipley MJ, et al. Alcohol and mortality: a U-shaped curve. Lancet 1981 ; i:580-3.

4 Friedman LA, Kimball AW. Coronary heart disease mortality and alcohol consumption in Framingham. Am J Epidemiol 1986;124:481-9.

5 Mukamal KJ, Maclure M, Muller JE, et al. Prior alcohol consumption and mortality following acute myocardial infarction. JAMA 2001;285:1965-70.

6 Muntwyler J, Hennekens CH, Buring JE, et al. Mortality and light to moderate alcohol consumption after myocardial infarction. Lancet 1998;352:1882-5.

7 Langer RD, Criqui MH, Reed DM. Lipoproteins and blood pressure as biological pathways for effect of moderate alcohol consumption on coronary heart disease. Circulation 1992;85:910-5.

8 Mennen LI, Balkau B, Vol S, et al. Fibrinogen: a possible link between alcohol consumption and cardiovascular disease? DESIR study group. Arterioscler Thromb Vasc Biol 1999:19:887-92.

9 Mukamal KJ, Jadhar PP, D'Agostino RB, et al. Alcohol consumption and hemostatic factors: analysis of the Framingham offspring cohort. Circulation 2001;104:1367-73

10 Imhof $A$, Froehlich $M$, Brenner $H$, et al. Effect of alcohol consumption on systemic markers of inflammation. Lancet 2001:357:763-7.

11 Kiechl S, Willeit J, Poewe W, et al. Insulin sensitivity and regular alcohol consumption: large, prospective, cross sectional population study. BMJ 1996;313:1040-4.

12 Cuevas AM, Guasch V, Castillo O, et al. A high-fat diet induces and red wine counteracts endothelial dysfunction in human volunteers. Lipids 2000;35:143-8.

13 Corder R, Douthwaite JA, Lees DM, et al. Endothelin-1 synthesis reduced by red wine. Nature 2001;414:863-4.

14 Nigdikar SV, Williams NR, Griffin BA, et al. Consumption of red wine polyphenols reduces the susceptibility of low-density lipoproteins to oxidation in vivo. Am J Clin Nutr 1998;68:258-65.

15 lijima K, Yoshizumi $M$, Hashimoto $M$, et al. Red wine polyphenols inhibit proliferation of vascular smooth muscle cells and downregulate expression of cyclin A gene. Circulation 2000;101:805-11.

16 Hendrickson RJ, Cahill PA, McKillop IH, et al. Ethanol inhibits mitogen activated protein kinase activity and growth of vascular smooth muscle cells in vitro. Eur J Pharmacol 1998;362:251-9.

17 Dekker JM, Schouten EG, Klootwiik P, et al. Heart rate variability from short electrocardiographic recordings predicts mortality form all causes in middleaged and elderly men. Am J Epidemiol 1997;145:899-908

18 Tsuji H, Venditti FJ, Manders ES, et al. Reduced heart rate variability and mortality risk in an elderly cohort: the Framingham heart study. Circulation 1994:90:878-83.

19 Kleiger RE, Miller JP, Bigger JT, et al. Decreased heart rate variability and its association with increased mortality after acute myocardial infarction. Am J Cardiol 1987;59:256-62.
20 Bigger JT, Fleiss JL, Steinman RC, et al. Frequency domain measures of heart period variability and mortality after myocardial infarction. Circulation 1992;85:164-71.

21 Qiuntana $M$, Storck N, Lindblad LE, et al. Heart rate variability as a means of assessing prognosis after acute myocardial infarction: a 3-year follow-up study. Eur Heart $J$ 1997; 18:789-97.

22 La Rovere MT, Bigger JT Jr, Marcus Fl, et al. Baroreflex sensitivity and heartrate variability in prediction of total cardiac mortality after myocardial infarction. ATRAMI (autonomic tone and reflexes after myocardial infarction) investigators. Lancet 1998;351:478-84.

23 Huikuri HV, Makikallio T, Peng CK, et al. Fractal correlation properties of RR interval dynamics and mortality in patients with depressed left ventricular function after an acute myocardial infarction. Circulation 2000;101:47-53.

24 Kupari M, Virolainen J, Koskinen P, et al. Short-term heart rate variability and factors modifying the risk of coronary artery disease in a population sample. Am J Cardiol 1993;72:897-903.

25 Tsuji H, Venditti FJ Jr, Manders ES, et al. Determinants of heart rate variability. J Am Coll Cardiol 1996:28:1539-46.

26 Christensen JH, Skou HA, Fog L, et al. Marine n-3 fatty acids, wine intake, and heart rate variability in patients referred for coronary angiography. Circulation 2001;103:651-7.

27 Ryan JM, Howes LG. Relations between alcohol consumption, heart rate, and heart rate variability in men. Heart 2002;88:641-2.

28 Virtanen R, Jula A, Kuusela T, et al. Reduced heart rate variability in hypertension: associations with lifestyle factors and plasma renin activity. $J$ Hum Hypertens 2003;17:171-9.

29 Stolarz K, Staessen JA, Kuznetsova T, et al. Host and environmental determinants of heart rate and heart rate variability in four European populations. J Hypertens 2003;21:525-35.

30 Burell G, Granlund B. Women's hearts need special treatment. Int J Behav Med 2002;9:228-42.

31 Nygårds ME. An automated system for ECG monitoring. Comput Biomed Res 1978;12:181-202.

32 Anon. Heart rate variability: standards of measurement, physiological interpretation and clinical use. Task force of the European Society of Cardiology and the North American Society of Pacing and Electrophysiology. Circulation 1996;93:1043-65.

33 Janszky I, Ericson M, Mittleman MA, et al. Heart rate variability in long-term risk assessment in middle aged women with coronary heart disease: the Stockholm female coronary risk study. J Intern Med 2004;255:13-21.

34 Willett WC, Sampson L, Stampfer MJ, et al. Reproducibility and validity of a semiquantitative food frequency questionnaire. Am J Epidemiol 1985; 122:51-65.

35 Larsson SC, Giovannucci E, Wolk A. Dietary folate intake and incidence of ovarian cancer: the Swedish mammography cohort. I Natl Cancer Inst 2004;96:396-402.

36 Rubin R. Effect of ethanol on platelet function. Alcohol Clin Exp Res 1999:23:1114-8.

37 Gronbaek M. Alcohol, type of alcohol, and all-cause and coronary hear disease mortality. Ann N Y Acad Sci 2002;957:16-20.

38 Rimm EB, Klatsky A, Grobbee D, et al. Review of moderate alcohol consumption and reduced risk of coronary heart disease: is the effect due to beer, wine or spirits? BMJ 1996;312:731-6.

39 Cleophas TJ. Wine, beer and spirits and the risk of myocardial infarction: a systematic review. Biomed Pharmacother 1999;53:417-23.

40 Koskinen P, Virolainen J, Kupari M. Acute alcohol intake decreases short-term heart rate variability in healthy subjects. Clin Sci (Lond) 1994;87:225-30.

41 Rossinen J, Viitasalo M, Partanen J, et al. Effects of acute alcohol ingestion on heart rate variability in patients with documented coronary artery disease and stable angina pectoris. Am J Cardiol 1997:79:487-91.

42 Bennett AJ, Sponberg AC, Graham T, et al. Initial ethanol exposure results in decreased heart rate variability in ethanol-naive rhesus monkeys. Eur J Pharmacol 2001;433:169-72.

43 Minami J, Todoroki M, Ishimitsu T, et al. Effects of alcohol intake on ambulatory blood pressure, heart rate, and heart rate variability in Japanese men with different ALDH2 genotypes. J Hum Hypertens 2002;16:345-51.

44 Malpas SC, Whiteside EA, Maling TJ. Heart rate variability and cardiac autonomic function in men with chronic alcohol dependence. $\mathrm{Br}$ Heart $\mathrm{J}$ $1991 ; 65: 84-8$.

45 DePetrillo PB, White KV, Liu M, et al. Effects of alcohol use and gender on the dynamics of EKG time-series data. Alcohol Clin Exp Res 1999;23:745-50.

46 Shaper AG, Wannamethee G, Walker M. Alcohol and mortality in British men: explaining the U-shaped curve. Lancet, 1988;ii, 1267-73.

47 Di Castelnuovo A, Rotondo S, lacoviello L, et al. Meta-analysis of wine and beer consumption in relation to vascular risk. Circulation 2002;105:2836-44.

48 Klatsky AL, Friedman GD, Armstrong MA, et al. Wine, liquor, beer, and mortality. Am J Epidemiol 2003;158:585-95.

49 Reynolds K, Lewis B, Nolen JD, et al. Alcohol consumption and risk of stroke: a meta-analysis. JAMA 2003;289:579-88. 